\title{
Relaxation of a three-level atom in stochastic fields and its spectroscopic properties
}

\author{
V.A. Mikhailov ${ }^{1, *}$, and N.V. Troshkin ${ }^{1}$ \\ ${ }^{1}$ Samara National Research University, 443086 Samara, Russia
}

\begin{abstract}
Relaxation of a motionless three-level atom with a nonequidistant spectrum in a photon heat bath and an external stochastic field is investigated. In the Markovian approximation of the relaxation processes a master equation for the reduced density matrix is derived by making use of the averaging over realizations of the stochastic field. The master equation is solved exactly. The correlation functions, the shapes of the radiation lines, the intensity correlation and photon number spectrums are calculated for the $V, \Xi$ and $\Lambda$ atoms and realizations of the stochastic field by a delta-correlated and a Kubo-Anderson processes.
\end{abstract}

Spectroscopic properties of relaxation of two-level systems driven by external stochastic fields were studied in $[1,2]$. Here the case of a three-level system is investigated by means of a master equation averaged over stochastic fields realizations.

The Hamiltonian of a motionless three-level atom with a non-equidistant spectrum interacting with a photon heat bath and an external stochastic field has the next form [3]:

$$
\hat{H}=\hat{H}_{A}+\hat{H}_{T}+\hat{H}_{A T}+\hat{H}_{s t} \text {, }
$$

where $\hat{H}_{A}-$ the Hamiltonian of an isolated atom, $\hat{H}_{T}-$ the photon heat bath thermostat, $\hat{H}_{A T}$ - the Hamiltonian of the interaction between the atom and the heat bath.

The Hamiltonian of the interaction between the atom and the stochastic field can be written as:

$$
\hat{H}_{s t}=\hbar\left(\Omega(t) \hat{H}_{1}+\Xi(t) \hat{H}_{2}\right)+\hbar\left(\xi(t) \hat{J}_{+}+\zeta(t) \hat{K}_{+}+\lambda(t) \hat{L}_{+}+\text {h.c. }\right),
$$

where $\hat{H}_{1}$ and $\hat{H}_{2}$ are diagonal operators defining energy levels of the atom; $\hat{J}_{ \pm}, \hat{K}_{ \pm}$and $\hat{L}_{ \pm}$ are operators of transitions between energy levels of the atom. The listed operators are generators of the $S U(3)$ group. $\Omega(t)$ and $\Xi(t)$ define random shifts of atomic energy levels; $\xi(t), \zeta(t)$ and $\lambda(t)$ are random functions proportional to intensity of the external stochastic field and affecting transitions between energy levels of the atom.

In the Markovian approximation of the relaxation processes a master equation for the reduced density matrix is derived by making use of the averaging over realizations of the stochastic field $[4,5,6]$ :

\footnotetext{
* Corresponding author: va mikhailov@mail.ru
} 


$$
\begin{aligned}
& \frac{\partial\langle\hat{\rho}\rangle}{\partial t}=K_{\Omega}\left(\hat{H}_{1}\langle\hat{\rho}\rangle \hat{H}_{1}-\hat{H}_{1}^{2}\langle\hat{\rho}\rangle\right)+K_{\Xi}\left(\hat{H}_{2}\langle\hat{\rho}\rangle \hat{H}_{2}-\hat{H}_{2}^{2}\langle\hat{\rho}\rangle\right)+ \\
& +\left(\eta_{1}+K_{\xi}\right)\left(\hat{J}_{-}\langle\hat{\rho}\rangle \hat{J}_{+}-\hat{J}_{+} \hat{J}_{-}\langle\hat{\rho}\rangle\right)+\left(\delta_{1}+K_{\xi}\right)\left(\hat{J}_{+}\langle\hat{\rho}\rangle \hat{J}_{-}-\hat{J}_{-} \hat{J}_{+}\langle\hat{\rho}\rangle\right)+ \\
& +\left(\eta_{2}+K_{\zeta}\right)\left(\hat{K}_{-}\langle\hat{\rho}\rangle \hat{K}_{+}-\hat{K}_{+} \hat{K}_{-}\langle\hat{\rho}\rangle\right)+\left(\delta_{2}+K_{\zeta}\right)\left(\hat{K}_{+}\langle\hat{\rho}\rangle \hat{K}_{-}-\hat{K}_{-} \hat{K}_{+}\langle\hat{\rho}\rangle\right)+ \\
& +\left(\eta_{3}+K_{\lambda}\right)\left(\hat{L}_{-}\langle\hat{\rho}\rangle \hat{L}_{+}-\hat{L}_{+} \hat{L}_{-}\langle\hat{\rho}\rangle\right)+\left(\delta_{3}+K_{\lambda}\right)\left(\hat{L}_{+}\langle\hat{\rho}\rangle \hat{L}_{-}-\hat{L}_{-} \hat{L}_{+}\langle\hat{\rho}\rangle\right)+\text { h.c. },
\end{aligned}
$$

where

$$
\begin{aligned}
& \eta_{j}=\frac{\gamma_{j}}{2}\left(N_{j}+1\right), \quad \delta_{j}=\frac{\gamma_{j}}{2} N_{j}, \quad j=1,2,3 ; \\
& K_{\Omega}=\int_{t_{0}}^{t}\left\langle\Omega(t) \Omega\left(t_{1}\right)\right\rangle d t_{1}, \quad K_{\Xi}=\int_{t_{0}}^{t}\left\langle\Xi(t) \Xi\left(t_{1}\right)\right\rangle d t_{1}, \quad K_{\xi}=\int_{t_{0}}^{t}\left\langle\xi(t) \bar{\xi}\left(t_{1}\right)\right\rangle d t_{1}, \quad K_{\zeta}=\int_{t_{0}}^{t}\left\langle\zeta(t) \bar{\zeta}\left(t_{1}\right)\right\rangle d t_{1}, \\
& K_{\lambda}=\int_{t_{0}}^{t}\left\langle\lambda(t) \bar{\lambda}\left(t_{1}\right)\right\rangle d t_{1},
\end{aligned}
$$

$\gamma_{j}$ is the damping constant for the $j$-th transition of the atom, $N_{j}$ is the average number of photons in the photon heat bath on the $j$-th transition of the atom.

The interaction of the atom with the external stochastic field is considered in the same order of smallness as the interaction of the atom with the photon heat bath.

Influence of model parameters of the stochastic field on shapes of radiation lines, spectrums of intensity correlations and number of photons is investigated for a deltacorrelated process and a Kubo-Anderson process.

\section{References}

1. R. Walser, P. Zoller, Phys. Rev. A 49, 5067 (1994)

2. I.A. Goychuk, Phys. Rev. E 51, 6267 (1995)

3. V.A. Mikhailov, N.V. Troshkin, Proc. SPIE 9917, 991731 (2016)

4. E.G. Petrov, Phys. Rev. E 57, 94 (1998)

5. V.A. Mikhailov, Theoretical Physics 7, 93 (2006)

6. V.A. Mikhailov, N.V. Troshkin, Computer Optics 40, 61 (2016) 